\title{
Poly (C)-Binding Protein 1: A Novel Function Attributed to the Nervous System Microenvironment
}

\author{
Bingbing Jia, Lirong Huo*, Meng Ye, Chensi Liang \\ Central Laboratory, Fuxing Hospital, The Eight Clinical Medical College, Capital Medical University, Beijing, China \\ Email: ^huolir@ccmu.edu.cn
}

How to cite this paper: Jia, B.B., Huo, L.R., Ye, M. and Liang, C.S. (2021) Poly (C)-Binding Protein 1: A Novel Function Attributed to the Nervous System Microenvironment. World Journal of Neuroscience, 11, 48-66.

https://doi.org/10.4236/wjns.2021.111006

Received: November 16, 2020

Accepted: February 22, 2021

Published: February 25, 2021

Copyright $\odot 2021$ by author(s) and Scientific Research Publishing Inc. This work is licensed under the Creative Commons Attribution International License (CC BY 4.0).

http://creativecommons.org/licenses/by/4.0/

(c) (i) Open Access

\begin{abstract}
Poly (C)-binding protein 1 (PCBP1), which acts as an RNA binding protein, has multiple functions and regulates gene expression by binding to polycytosine, poly $(\mathrm{C})$. The aim of the present study was to investigate the novel function of PCBP1 in the nervous system microenvironmen. The overexpression of PCBP1 has a critical effect on the proliferation and anti-apoptosis of neuroblastoma and glial cells in the direct model, whereas the overexpression of PCBP1 in neuroblastoma or glial cells cannot affect the proliferation or apoptosis of neuroblastoma or glial cells through substances secreted extracellularly. Furthermore, through direct or indirect actions, PCBP1 suppressed the expression of nuclear factor kappa B (NF- $\kappa$ B) and the inflammatory cytokine interleukin (IL)-6 and increased the levels of the anti-inflammatory cytokine IL-10; additionally, PCBP1 changed the expression of functional proteins, such as heat shock protein 70 (HSP70), glial cell line-derived neurotrophic factor (GDNF) and brain-derived neurotrophic factor (BDNF). The results suggest that PCBP1 can regulate the nervous system in proliferation, apoptosis, inflammatory response and expression of relevant functional proteins, and it could provide novel targets for gene treating human neurological disorders.
\end{abstract}

\section{Keywords}

PCBP1, Neurological Disorders, Inflammatory, Proliferation, Apoptotic

\section{Introduction}

Poly (C)-binding protein 1 (PCBP1), also known as aCP1 or hnRNP E1, is a bridge protein with multiple biological functions in mammalian cells [1]. PCBP1 
belongs to a subfamily of heterogeneous nuclear ribonuclear proteins (hnRNPs), and this subfamily contains three hnRNP $\mathrm{K}$ homology $(\mathrm{KH})$ domains. PCBP1 regulates gene expression through the $\mathrm{KH}$ structural domain interaction with single- and double-stranded DNA [2] [3]. As a signal cofactor, PCBP1 has been implicated in multiple functions, such as DNA replication, transcriptional regulation, posttranslational modifications and mRNA translation and stabilization [4] [5] [6].

Perhaps most importantly, PCBP1 can participate in the interactions of proteins with nucleic acids or proteins. It was found that an AU-rich element (ARE) is recognized by PCBP1, although several PCBP1-binding sites are CU-rich elements or oligo ( $\mathrm{rC}$ ) elements [7] [8] [9]. Recent studies indicated that PCBP1 could regulate alternative splicing of the $\mathrm{CD} 44$ gene and act as global regulators of alternative polyadenylation [10]. It has been reported that a loss of phosphorylation regulation of PCBP1 expression or posttranslational modification is associated with carcinoma or metastasis, such as non-small-cell lung cancer (NSCLC), thyroid carcinoma, and prostate cancer [11] [12] [13]. PCBP1 can also transport iron through metal regulation and protein interaction, and there are four main targets: ferritin, prolyl hydroxylases (PHDs), asparaginyl hydroxylase (FIH1) and deoxyhypusine hydroxylase (DOHH) [14] [15] [16]. In addition, PCBP1 also functions as a negative regulator of vesicular stomatitis virus (VSV) to inhibit the expression of virus genes [17].

Nuclear factor kappa B (NF- $\kappa$ B), which is a sensor of oxidative stress and is important for inflammatory reactions [18], modulates the expression of both anti-apoptotic and apoptotic genes [19] and accumulates in dopaminergic neurons in neurodegenerative diseases, such as Parkinson's disease and Alzheimer's disease [20]. NF- $\kappa \mathrm{B}$ is composed of $50 \mathrm{kDa}$ (p50) and $65 \mathrm{kDa}(\mathrm{p} 65)$ heterodimer subunits. It has been reported that NF- $\kappa \mathrm{B}$ (p65) can be expressed in neurons of the striatum, substantia nigra and hippocampus. A previous interesting study indicated that the overexpression of PCBP1 can augment the signal transducer and activator of transcription 3 (STAT3)-mediated suppression of NF- $\kappa \mathrm{B}$ activation and enhance the antagonistic effect of IL-10 on IL- 6 production, while inhibition of PCBP1 reduces it [21].

Intriguingly, in addition to their canonical roles in the above study, some studies gradually uncovered some functions of PCBP1 in nervous system diseases. Previously, we found that PCBP1 can mediate mRNA transfer through the interaction of fibrillar layer proteins $\mathrm{A} / \mathrm{C}$ in Gilford progeria syndrome [22]. A recent study uncovered that PCBP1 has a greatly increased interaction with the small heat shock protein HSPB1 (HSP27), which results in a reduction of translational repressive activity in neurodegenerative disease [23]. In our previous study, when the endogenous PCBP1 gene is knocked down, many genes related to functions such as neuron occurrence, development, and axon formation are affected [24]. Thus far, these studies have indicated that PCBP1 might provide neuroprotection by promoting neurodevelopment. However, how PCBP1 promotes neuroprotection in the neural system microenvironment has not been ex- 
tensively studied.

This study shows that PCBP1 is closely related to the proliferation, anti-apoptosis, anti-inflammatory and neurotrophic factor secretion of neuroblastoma cells and glial cells, suggesting that PCBP1 plays a significant role in the nervous system microenvironment. In this study, it was further confirmed that PCBP1 could increase the expression of GDNF, BNDF and HSP70, and the enhanced expression was consistent with the proliferation of neurons and glial cells. The results suggest that, to some extent, PCBP1 can provide a good living microenvironment for nerve cells and glial cells and plays a crucial role in the occurrence and development of neurological diseases.

\section{Materials and Methods}

\section{Cell culture}

The human neuroblastoma cell line (SH-SY5Y) was cultured in RMPI-1640 medium (Thermo Fisher, Milan, Italy), the microglial cell line (BV-2) was cultured in DMEM/F-12 medium (Thermo Fisher), and the human astrocyte cell line (HA) was cultured in astrocyte medium (ScienCell, San Diego, California, USA). All media were supplemented with $10 \%$ fetal bovine serum (Thermo Fisher) and $1 \%$ penicillin-streptomycin antibiotics (Thermo Fisher). Cells were cultured at $37^{\circ} \mathrm{C}$ with $5 \% \mathrm{CO}_{2}$ in an incubator.

\section{Transfection of plasmids}

SH-SY5Y, BV-2 and HA cells were seeded in six-well plates and cultured overnight prior to transfection. Plasmid transfections were conducted by either mixing cell culture and plasmid or Opti-MEM (Thermo Fisher) and Lipofectamine 2000 Reagent (Thermo Fisher) at room temperature and incubating for 5 min. Then, the plasmid solution and the transfection reagent were mixed and incubated for an additional 20 min before being added to cells. The medium was changed $8 \mathrm{~h}$ posttransfection. The overexpression efficiency of PCBP1 was determined by Western blotting and real-time PCR.

\section{The direct and indirect action models of PCBP1}

For the direct action model (Figure 1(A)), control plasmid pEGFP/N1 and recombinant plasmid pEGFP/N1-PCBP1 were transfected into SH-SY5Y, BV-2 and HA cells, according to the above method. The cell supernatant was returned $48 \mathrm{~h}$ after transfection and was stored in $-80^{\circ} \mathrm{C}$.

For the indirect model (Figure 1(B)), SH-SY5Y, BV-2 and HA cells were seeded in a cell culture plate. After cells were cultured for $24 \mathrm{~h}$, the cell culture media was removed, and HA, BV-2 and SH-SY5Y cells were continually incubated for $48 \mathrm{~h}$ in complete media containing the supernatant of cells transfected with pEGFP/N1-PCBP1 and pEGFP/N1 and fresh complete media (1:2 ratio). The indirect action model was carried out in two different ways: 1 ) for the effects of neurons on glial cells (represented as SH-SY5Y--BV-2 and SH--SY5Y-HA), the culture media of SH-SY5Y transfected with pEGFP/N1-PCBP1 and pEGFP/N1 was added into the media of BV-2 and HA cells for $48 \mathrm{~h}$ or 2) for the effect of glial cells on neurons (represented as BV-2-SH-SY5Y and HA--SH-SY5Y), the 


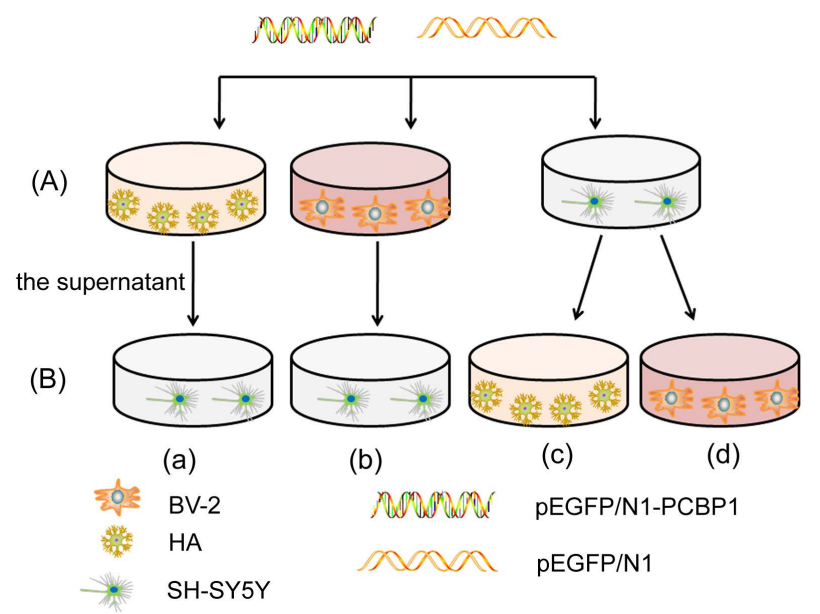

Figure 1. Graphical schematic of the experimental setup. (A) The direct action model: HA, BV-2 and SH-SY5Y cells were seeded in cell culture plates and transfected with pEGFP/N1 and pEGFP/N1-PCBP1 for 48 h. (B) The indirect model: SH-SY5Y, BV-2 and $\mathrm{HA}$ cells were seeded in cell culture plates and incubated for $48 \mathrm{~h}$ in complete media containing the pEGFP/N1-PCBP1 and pEGFP/N1 supernatants and fresh complete media at a 1:2 ratio. The effects of glial cells on neurons were represented as HA--SH-SY5Y (a) and BV-2--SH-SY5Y (b); the effects of neurons on glial cells were represented as SH-SY5Y--HA (c) and SH-SY5Y--BV-2 (d).

culture media of BV-2 and HA cells transfected with pEGFP/N1-PCBP1 and pEGFP/N1 was added into the media of SH-SY5Y cells for $48 \mathrm{~h}$. Supernatants were collected for ELISA analysis and the samples in the direct and indirect models were prepared for CCK-8, cell count, real-time PCR, flow cytometry, Western-blotting and confocal laser scanning microscopy (CLSM).

\section{Cell proliferation assay}

A total of $5 \times 10^{3}$ SH-SY5Y, BV-2 or HA cells were seeded in 96-well plates with transfection or cell supernatant stimulation. After time-course incubations, the culture medium in each well was removed and washed three times with phosphate buffer saline (PBS). Then, $10 \mu \mathrm{L}$ CCK-8 solution (Sangon Biotech, Shanghai, China) was added and incubated for $2.5 \mathrm{~h}$ at $37^{\circ} \mathrm{C}$. The absorbance was detected at $450 \mathrm{~nm}$ using a microplate reader (Thermo Fisher).

\section{Apoptosis analysis}

An AnnexinV-PE Apoptosis Detection Kit (Beyotime, Beijing, China) and an Annexin V-FITC Apoptosis Detection Kit (Sangon Biotech) were used to stain apoptotic cells. SH-SY5Y, HA and BV-2 cells were seeded in 6-well plates at $1 \times$ $10^{5}$ cells/well with transfection or cell supernatant stimulation and were continuously cultured for $48 \mathrm{~h}$. Then, the cells in each well were collected by $0.25 \%$ EDTA-free trypsin and resuspended in $200 \mu \mathrm{L}$ Binding Buffer containing $10 \mu \mathrm{L}$ Annexin V-PE or Annexin V-FITC. Following incubation at room temperature for $10 \mathrm{~min}$ in the dark, $5 \mu \mathrm{L}$ PI was added, and the samples were immediately analyzed by flow cytometry (Becton Dickinson FACS Calibur, Franklin lake, New Jersey, USA).

Enzyme-linked Immunosorbent Assay (ELISA) 
ELISA kits for mouse IL-6, mouse IL-10, human IL-10 and human IL-6 were purchased from multiscience (Hangzhou, China). First, $100 \mu \mathrm{L}$ 2-fold diluted standard and $100 \mu \mathrm{L}$ samples with appropriate dilutionswere added to the coated plate. Secondly, $50 \mu \mathrm{L}$ diluted primary antibody was added to each well and incubated for $1.5 \mathrm{~h}$ at $25^{\circ} \mathrm{C}$ followed by washing 6 times and addition of $100 \mathrm{uL}$ antibody conjugated with streptavidin-horseradish peroxidase. After the sample was incubated for $30 \mathrm{~min}$ at $25^{\circ} \mathrm{C}, 100 \mathrm{uL}$ substrate solution was added to each well and the plate was measured at $450 \mathrm{~nm}$.

\section{Confocal laser scanning microscopy (CLSM)}

SH-SY5Y, BV-2, and HA cells were dropped on sterile cell slides in a 6-well cell plate. After the cells were stably expressing pEGFP/N1-PCBP1 and pEGFP/N1 or were stimulated with cell supernatant for $48 \mathrm{~h}$, the supernatant was removed, fixed with $4 \%$ paraformaldehyde for $15 \mathrm{~min}$ at room temperature, and then the excess fixative was washed off with $1 \times$ PBS; then, $0.2 \%$ Triton $\mathrm{X}-100 / 1 \times \mathrm{PBS}$ was added and incubated at $37^{\circ} \mathrm{C}$ for $15 \mathrm{~min}$. Slides were washed with $1 \times \mathrm{PBS}$ and $0.1 \%$ Triton $\mathrm{X}-100 / 1 \times$ PBS twice for 5 min each time and blocked in $10 \%$ FBS/1 $\times$ PBS at room temperature for $45 \mathrm{~min}$; coverslips were incubated with primary antibodies ( (anti-HSP70 mouse (1:50; Santa Cruz Biotechnology, Dallas, USA, \# sc-373867), anti-BDNF mouse (1:50; Santa Cruz \# sc-65514), anti-NF- $\kappa$ B p65 mouse (1:50; Santa Cruz \# sc-8008), anti-GDNF mouse (1:50; Santa Cruz \# sc-13147)) at $4^{\circ} \mathrm{C}$ overnight. The next day, Alexa Fluor 594-conjugated goat anti-mouse IgG $(\mathrm{H}+\mathrm{L})$ (ZSGB-BIO, Beijing, China, \#ZF-0513) or Alexa Fluor 48 -conjugated goat anti-mouse IgG $(\mathrm{H}+\mathrm{L})$ (ZSGB-BIO \#ZF-05127) dissolved in $10 \% \mathrm{FBS}$ at a 1:50 dilution was dropped onto each coverslip and incubated for $1 \mathrm{~h}$. Finally, nuclei were counterstained with a solution of Hoechst 33,258 (Beyotime \#C1017) for $10 \mathrm{~min}$ at room temperature, and then, coverslips were sealed.

\section{Quantitative real-time PCR analysis}

Total RNA was extracted using TRIzol Reagent (Thermo Fisher) according to the manufacturer's protocol. One microgram of RNA was reverse transcribed for cDNA synthesis. Real-time quantification was carried out in triplicate with Power SYBR Green PCR Master Mix (Applied Biosystems, Carlsbad, CA, USA) on a StepOnePlus Real-Time PCR System (Thermo Fisher). The cycle rection was carried out according to the manufacturer's instructions. The following primers were used: GAPDH-F: 5'-ATCAGCAATGCCTCCTGCAC-3'; GAPDH-R: 5'-TGGTCATGAGTCCTTCC ACG-3'; PCBP1-F: 5'-TTCTAGAATTCATGGATGCCGGTGTGACTGAAAGTG-3'; PCBP1-R: 5'-TTCTAGAATTCAACCTACACTGTTCTAGCTGCACC-3'. The results were analyzed according to the $2^{(-\Delta \Delta \mathrm{Ct})}$ method. Three independent experiments were carried out for each assay condition.

\section{Western blot analysis}

Protein extracts were made using RIPA buffer, and soluble proteins were separated by SDS-PAGE and blotting onto a PVDF membrane (Millipore, Milan, Italy). Membranes were blocked using $5 \%$ milk powder (in $1 \times \mathrm{T}$-TBS buffer), 
followed by incubation with primary antibodies: (anti-HSP70 mouse (1:100; Santa Cruz \#sc-373867), anti-BDNF mouse (1:100; Santa Cruz \#sc-65514), anti-NF- $\kappa$ B p65 mouse (1:100; Santa Cruz \#sc-8008), anti-GDNF mouse (1:100; Santa Cruz \#sc-13147), anti-hnRNP E1 mouse (1:100; Santa Cruz \#sc-137249), anti-GAPDH rabbit (1:100; Cell Signaling Technology, Danvers, MA, USA, \#5174) overnight at $4^{\circ} \mathrm{C}$. After washing 3 times with $1 \times \mathrm{T}$-TBS buffer, secondary antibodies were applied for $1 \mathrm{~h}$ at room temperature. Finally, protein signals were detected by Super Signal West Pico Chemiluminescent Substrate (Thermo Fisher) and observed in a dark room. GAPDH served as a loading control, and the data were presented as a relative fold change using NIH Image J analysis software.

\section{Statistical analysis}

Data are presented as the mean of at least three experiments \pm standard deviation (SD). The results are recorded using Graphpad Prism 5 software (Graph Pad, San Diego, CA, USA). All statistical tests were performed using SPSS 20.0. Two-tailed $t$-tests or ANOVA analyses were used to estimate $\mathrm{p}$ values. $\mathrm{p}<0.05$ showed statistically significant differences.

\section{Results}

PCBP1 directly promotes the growth of SH-SY5Y, BV-2 and HA cells without any indirect effect on the growth of the cells.

To explore the direct and indirect effects of PCBP1 on the growth of SH-SY5Y, BV-2 and HA cells, recombinant plasmid pEGFP/N1-PCBP1 and control plasmid pEGFP/N1 were transfected into SH-SY5Y, BV-2 and HA cells using liposome-mediated transient transfection. After transfection for $48 \mathrm{~h}$, real-time qPCR and western blot revealed that the expression levels of PCBP1 in SH-SY5Y, BV-2 and HA cells were significantly increased by pEGFP/N1-PCBP1 when compared to pEGFP/N1 (Figure 2).

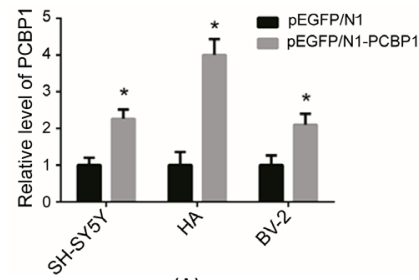

(A)

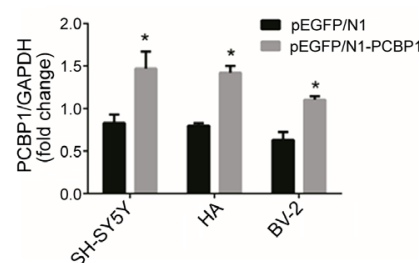

(B)

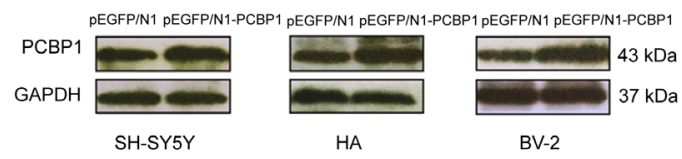

(C)

Figure 2. In the direct action model, Real-time qPCR and western-blot analyzed the SH-SY5Y, HA and BV-2 cells transfected with PCBP1. (A) Real-time qPCR and (B) western-blot revealed the different expression levels of PCBP1 in SH-SY5Y, HA and BV-2 cells transfected with pEGFP/N1 and pEGFP/N1-PCBP1. * Statistically significant differences $(\mathrm{p}<0.05)$ between $\mathrm{pEGFP} / \mathrm{N} 1$ and $\mathrm{pEGFP} / \mathrm{N} 1-\mathrm{PCBP} 1$.The bars express as mean \pm S.D., $\mathrm{n}=3$. 
Proliferation of SH-SY5Y, BV-2 and HA cells through the regulation of PCBP1 was detected by CCK- 8 and cell counting. First, a cell counting assay demonstrated that the number of SH-SY5Y and HA cells was obviously higher with pEGFP/N1-PCBP1 than in the control group from 1 - 8 days after transfection with pEGFP/N1-PCBP1 and pEGFP/N1 (Figure 3(A1) and Figure 3 (B1)). At 1 - 5 days after transfection, BV-2 cells transfected with pEGFP/N1-PCBP1 showed accelerated growth compared with the control group. However, at $6-8$ days after transfection, there was no significant difference in the number of BV-2 cells between the two groups (Figure $3(\mathrm{C} 1)$ ). We conducted a cell proliferation assays from 12 to $72 \mathrm{~h}$ after transfection of pEGFP/N1-PCBP1 and pEGFP/N1. The growth of SH-SY5Y, HA and BV-2 cells was obviously enhanced by pEGFP/N1-PCBP1 compared to pEGFP/N1 (Figure 3(A2) and Figure $3(\mathrm{C} 2)$ ).

We further observed whether PCBP1 had an indirect effect on the proliferation of SH-SY5Y, BV-2 and HA cells by a cell counting and CCK-8 assay. The

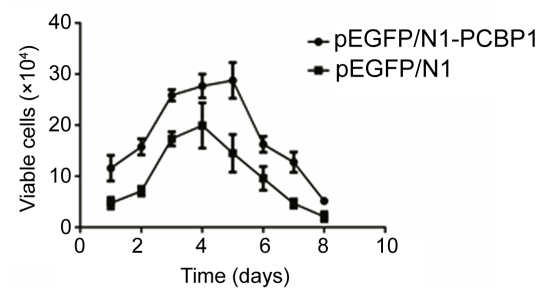

(A1)

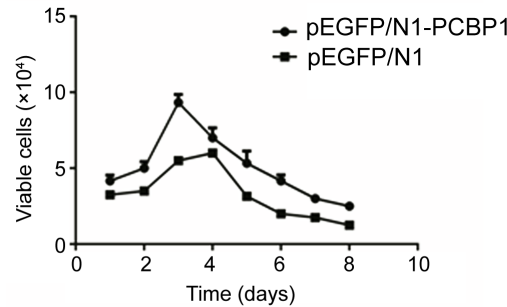

(B1)

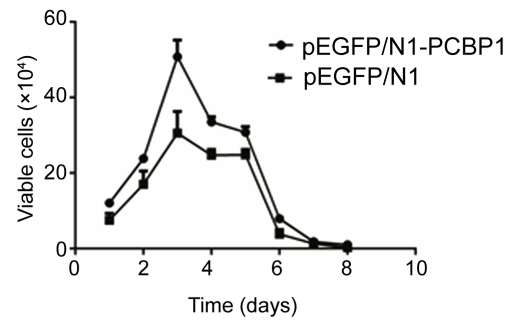

(C1)

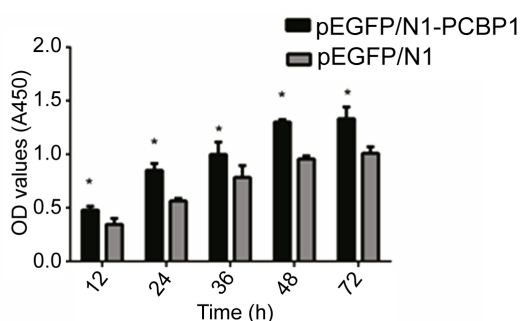

(A2)

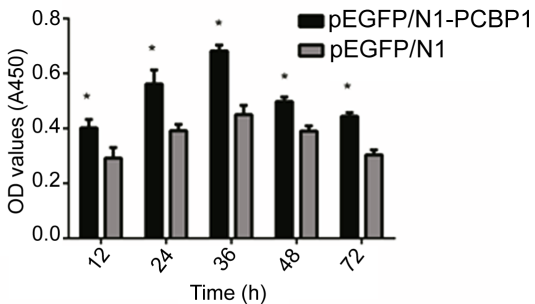

(B2)

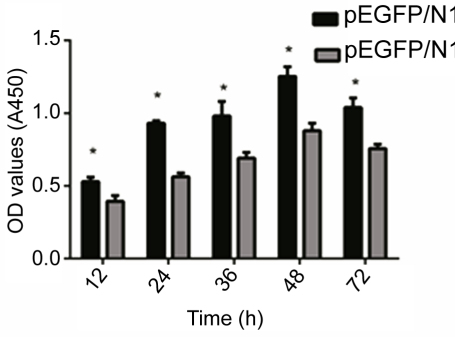

(C2)

Figure 3. In the direct action model, PCBP1 promotes the growth of SH-SY5Y, BV-2 and HA cells.CCK-8 was performed to study the cell vitality of SH-SY5Y, HA and BV-2. ((A1)-(C1)) Cell counting revealed the growth curve of the SH-SY5Y (A1), HA (B1) and BV-2 (C1) cells transfected with pEGFP/N1 and pEGFP/PCBP1 for 8 days. ((A2)-(C2)) CCK-8 revealed the growth of SH-SY5Y (A2), HA (B2) and BV-2 (C2) cells transfected with pEGFP/N1 and pEGFP/PCBP1 for $72 \mathrm{~h}$. * Statistically significant differences ( $\mathrm{p}<$ 0.05 ) between $\mathrm{pEGFP} / \mathrm{N} 1$ and $\mathrm{pEGFP} / \mathrm{PCBP} 1$. The bars express as mean \pm S.D., $\mathrm{n}=3$. Analysis was performed using Two-way ANOVA. 
growth of SH-SY5Y, BV-2 and HA cells was not significantly different $(\mathrm{p}>0.05)$ when comparing the cell supernatant containing the control plasmid to recombinant plasmid pEGFP/N1-PCBP1. According to the above data, PCBP1 has a direct stimulating effect on cell proliferation, whereas the production of PCBP1 overexpression has no significant effect on the growth of SH-SY5Y, BV-2 and HA cells.

PCBP1 has a direct inhibitory effect on the early apoptosis of SH-SY5Y, BV-2 and HA cells without significant indirect effects.

We next determined the role of PCBP1 in the early apoptosis of SH-SY5Y, BV-2 and HA cells. In the direct model, SH-SY5Y, BV-2 and HA cells transfected with pEGFP/N1-PCBP1 and pEGFP/N1 were further cultured for $48 \mathrm{~h}$, and apoptosis was detected by staining with Annexin V-PE/7-AAD. Compared with that of the control group, the early apoptosis rate of, HA and BV-2 cells transfected with pEGFP/N1-PCBP1 was significantly decreased, and the values were $55.390 \% \pm 3.291 \%$ VS $45.017 \% \pm 1.026 \%$ (SH-SY5Y), $18,583 \% \pm 2.718 \%$ VS $9,260 \% \pm 1.794 \%(\mathrm{HA})$ and $18.550 \% \pm 2.014 \%$ VS $8.720 \% \pm 1.197 \%(\mathrm{BV}-2)$ respectively (Figure 4).

In addition, we examined the indirect effects of PCBP1 on the early apoptosis of SH-SY5Y, BV-2 and HA cells by Annexin V-FITC/PI double staining. The supernatant of SH-SY5Y cell culture overexpressing PCBP1 did not have a statistically significant effect on the early apoptosis rate of BV-2 and HA cells compared to the control group. Similarly, the BV-2 and HA cell culture supernatants overexpressing PCBP1 did not have a statistically significant effect on the early apoptosis rate of SH-SY5Y cells. Taken together, PCBP1 only has direct anti-apoptotic effects on SH-SY5Y, HA and BV-2 cells, while PCBP1 cannot inhibit cell apoptosis by indirect action.

PCBP1 can play both direct and indirect roles in inhibiting IL-6 secretion and promoting the secretion of the anti-inflammatory factor IL-10.

Because neuron and glial cells can produce cytokines, we examined each of the inflammatory cytokines in the direct and indirect action models of PCBP1 and determined the gene expression of IL-6 and IL-10. In the direct action model, an ELISA assay indicated that there was a lesser degree of IL-6 in BV-2, HA and SH-SY5Y cells transfected with pEGFP/N1-PCBP1 compared with the control group, and the values were $161 \pm 6.658$ VS $784.055 \pm 12.386$ (BV-2), 780.223 \pm 5.608 VS $961.563 \pm 0.778(\mathrm{HA})$ and $644.675 \pm 39.671$ VS $779.024 \pm 2.458$ (SH-SY5Y) respectively (Figure 5(A)). The gene expression of IL-10 in the BV-2 cells transfected with pEGFP/N1-PCBP1 (395.672 \pm 4.884$)$ was a drastic increase compared with the control group (318.954 \pm 15.537$)$ (Figure 5(D)). In addition, the gene expression of IL-10 was not statistically significant in HA and SH-SY5Y with pEGFP/N1-PCBP1 and pEGFP/N1 (Figure 5(E)).

Our second model of indirect action involved culturing HA, BV-2 and SH-SY5Y cells for $48 \mathrm{~h}$ after the addition of the supernatant from cells transfected with pEGFP/N1-PCBP1 and pEGFP/N1 and fresh culture media. The 


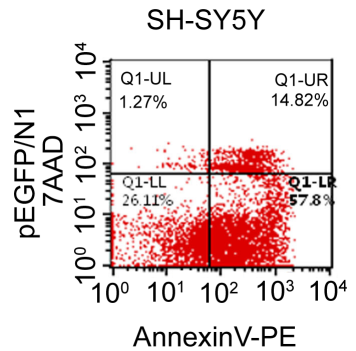

(A1)

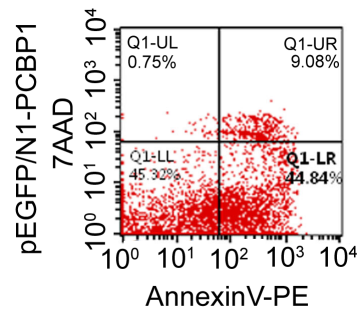

(A2)

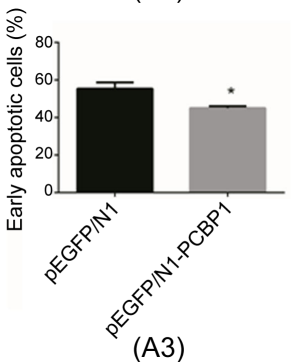

HA

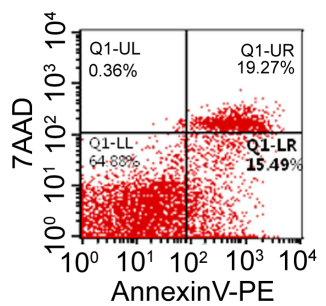

(B1)

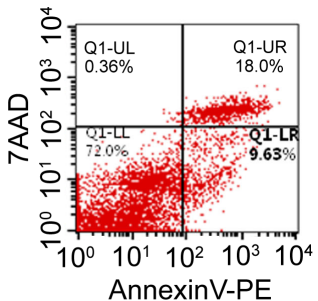

(B2)

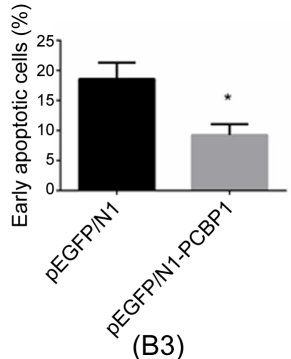

BV-2

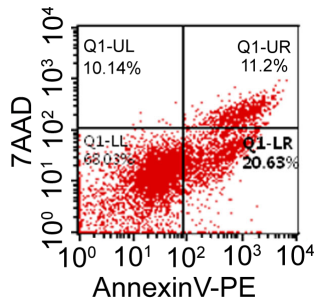

(C1)

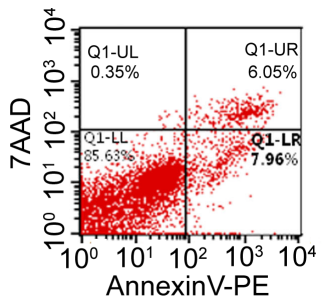

(C2)

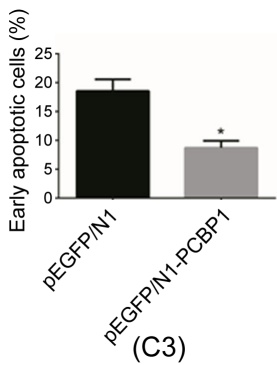

Figure 4. Flow cytometry analysis of SH-SY5Y, BV-2 and HA cells transfected with PCBP1 for $48 \mathrm{~h}$. (A1-A3) Annexin V-PE and 7-AAD staining and flow cytometry were used to assay the percentages of early apoptosis in the SH-SY5Y cells transfected with pEGFP/N1 and pEGFP/PCBP1. (B1-B3) Annexin V-PE and 7-AAD staining and flow cytometry were used to assay the percentages of early apoptosis in the HA cells transfected with pEGFP/N1 and pEGFP/PCBP1. (C1-C3) Annexin V-PE and 7-AAD staining and flow cytometry were used to assay the percentages of early apoptosis in the BV-2 cells transfected with pEGFP/N1 and pEGFP/PCBP1. * Statistically significant differences $(\mathrm{p}<$ 0.05 ) between pEGFP/N1 and pEGFP/PCBP1 in the SH-SY5Y (A), HA (B) and BV-2 (C) cells. The bars express as mean \pm S.D., $n=3$. (Q1-LR, the percentages of early apoptosis).

indirect action of PCBP1 generated a decline in the gene expression of IL-6 in $\mathrm{HA}$ and BV-2 and cells, and and the values were 453.209 \pm 10.210 VS 599.747 \pm 9.089 (SH-SY5Y--HA), $815.000 \pm 29.614$ VS $1217.667 \pm 60.451$ (SH-SY5Y--BV-2), $755.685 \pm 13.497 \mathrm{VS} 802.958 \pm 4.194(\mathrm{HA}--\mathrm{SH}-\mathrm{SY} 5 \mathrm{Y})$ and $653.075 \pm 10.302 \mathrm{VS}$ $776.481 \pm 11.587$ (BV-2--SH-SY5Y) respectively in the pEGFP/N1-PCBP1-transfected group compared to the control group (Figure $5(\mathrm{~B})$ ). There was a notable enhancement in IL-10 levels in BV-2 cells (306.354 \pm 17.352$)$ compared to that of the control group $(356.714 \pm 24.017)$, while the levels of IL-10 in HA and SH-SY5Y cells were not significantly different. (Figure 5(C) and Figure 5(D)). Our results indicate that PCBP1 in the direct and indirect action models reduced the levels of inflammatory cytokine IL-6 in HA, BV-2 and SH-SY5Y cells and increased the anti-inflammatory cytokine IL-10 in BV-2 cells, whereas there was no significant effect on IL-10 in HA and SH-SY5Y cells. 


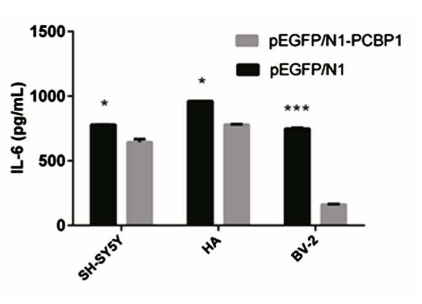

(A)

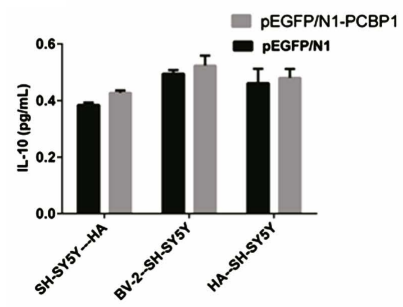

(C)

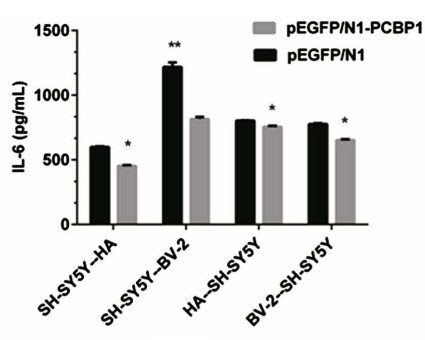

(B)

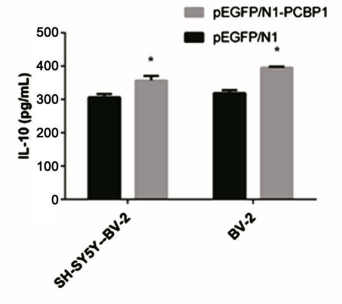

(D)

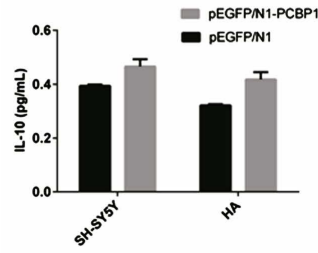

(E)

Figure 5. PCBP1 can play both direct and indirect roles in inhibiting IL-6 secretion and promoting the secretion of the anti-inflammatory factor IL-10. (A) Quantification of IL-6 using ELISA showed the decline of IL- 6 in the SH-SY5Y, HA and BV-2 cells transfected with $\mathrm{pEGFP} / \mathrm{N} 1$ and $\mathrm{pEGFP} / \mathrm{PCBP} 1$. Statistically significant differences $\left({ }^{*} \mathrm{p}<0.05,{ }^{* *} \mathrm{p}<\right.$ $\left.0.01,{ }^{* * *} \mathrm{p}<0.001\right)$ between $\mathrm{pEGFP} / \mathrm{N} 1$ and $\mathrm{pEGFP} / \mathrm{PCBP} 1$ in the SH-SY5Y, HA and BV-2 cells. (B) Quantification of IL-6 using ELISA showed the decline in IL-6 in the indirect action model. Statistically significant differences $\left({ }^{*} \mathrm{p}<0.05,{ }^{* *} \mathrm{p}<0.01\right)$ between pEGFP/N1 and pEGFP/PCBP1 in the SH-SY5Y, HA and BV-2 cells. (D) Quantification of IL-10 using ELISA showed the increased levels of IL-10 in the BV-2 cells and the indirect action of SH-SY5Y--BV-2. * Statistically significant differences $(\mathrm{p}<0.05)$ between pEGFP/N1 and pEGFP/PCBP1 in the BV-2 and SH-SY5Y--BV-2 cells. ((C), (E)) Quantification of IL-10 using ELISA showed no significant differences compared to control in the SH-SY5Y, HA cells (C) and the indirect action of SH-SY5Y--HA, BV-2--SH-SY5Y and HA--SH-SY5Y (E). The bars express as mean \pm S.D., $\mathrm{n}=3$.

\section{PCBP1 enhances HSP70, BDNF and GDNF expression and inhibits NF- $\kappa$ B} signaling pathway activation by direct and indirect effects.

First, we examined whether PCBP1 overexpression could regulate the expression of numerous genes in HA, BV-2, and SH-SY5Y cells, including NF- $\kappa \mathrm{B}$, HSP70, BDNF, and GDNF. pEGFP/N1-PCBP1 and pEGFP/N1 were transfected into HA, BV-2 and SH-SY5Y cells for $48 \mathrm{~h}$. Confocal laser scanning microscopy (CLSM) revealed that the fluorescence intensity (red color due to Alexa Fluor 594 fluorophore) of HSP70 in SH-SY5Y, HA and BV-2 cells was significantly increased in the pEGFP/N1-PCBP1-transfected group compared to the control group. In contrast, the fluorescence intensity of NF- $\kappa \mathrm{B}$ was relatively decreased in SH-SY5Y, HA and BV-2 and cells transfected with pEGFP/N1-PCBP1 compared to the control group (Figures 6(A)-(C)). The analysis of CLSM images revealed that the BV-2 and HA cells transfected with pEGFP/N1-PCBP1 exhibited higher expression of the GDNF gene than the pEGFP/N1 control. In the SH-SY5Y cells, the fluorescence intensity of BDNF was obviously enhanced by pEGFP/N1-PCBP1 when compared with the control group (Figures 6(A)-(C)). 


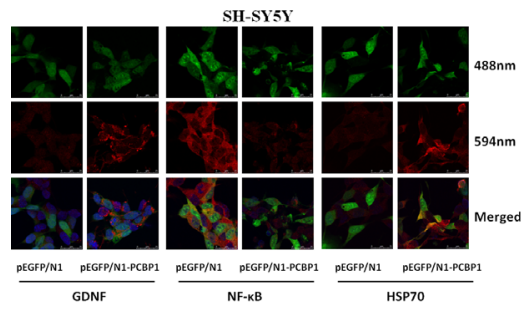

(A)

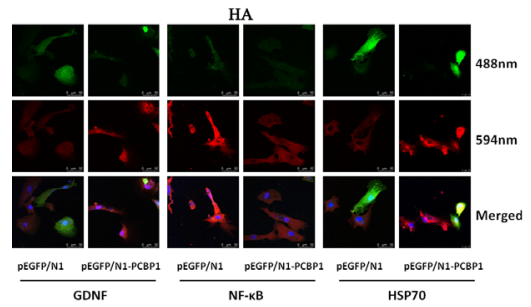

(B)

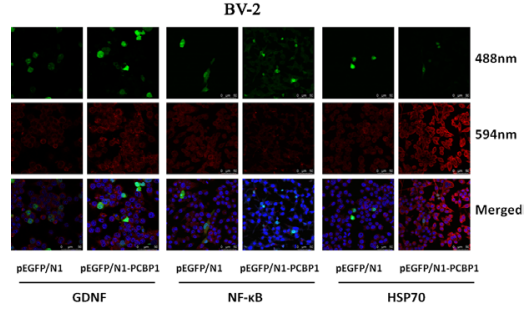

(C)

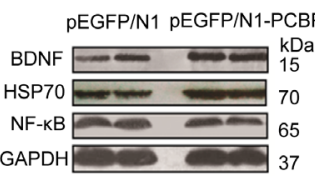

SH-SY5Y

(D1)

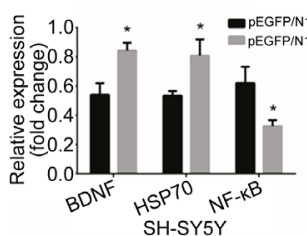

(D2)

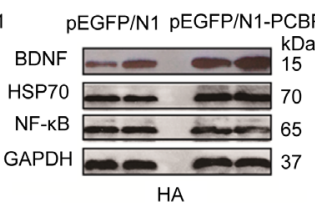

(E1)

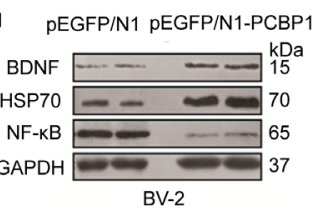

(F1)

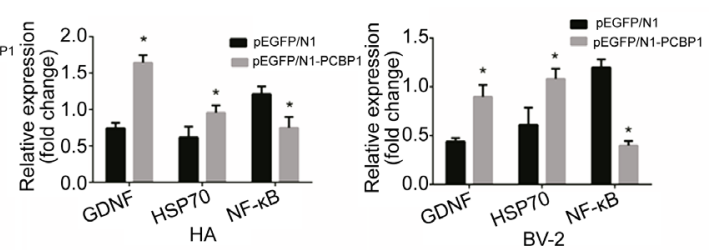

(E2)

(F2)

Figure 6. PCBP1 enhances HSP70, BDNF and GDNF expression and inhibits NF- $\kappa$ B signaling pathway activation by direct effects. In the confocal images, the top- and middle-rows show the transfection of pEGFP/N1 and pEGFP/N1-PCBP1, stained green, was observed at $488 \mathrm{~nm}$, and the fluorescence of HSP70, BDNF, GDNF and NF- $\kappa \mathrm{B}$, stained red, was observed at $594 \mathrm{~nm}$. In the bottom row, the images of the top and middle rows have been merged to obtain a composite. ((A)-(C)) Confocal images of SH-SY5Y (A), HA (B) and BV-2 (C) cells showed the transfection of pEGFP/N1 and pEGFP/PCBP1 (green), the increased fluorescence of HSP70, BDNF and GDNF (red), and the decreased fluorescence of NF- $\kappa$ B (red). ((D)-(F)) Western blot analysis showed increased levels of PCBP1, HSP70, BDNF and GDNF and decreased levels of NF- $\kappa$ B in SH-SY5Y ((D1) and (D2)), HA ((E1) and (E2)) and BV-2 ((F1) and (F2)) cells transfected with pEGFP/N1 and pEGFP/N1-PCBP1. * Statistically significant differences $(\mathrm{p}<0.05)$ between $\mathrm{pEGFP} / \mathrm{N} 1$ and pEGFP/N1-PCBP1. GAPDH was probed as a protein loading control for all experiments. The bars express as mean \pm S.D., $\mathrm{n}=3$. 
Simultaneously, we conducted Western blots to demonstrate that the proteins of HSP70, BDNF, and GDNF were significantly increased by pEGFP/N1-PCBP1, but the protein of NF- $\kappa$ B was downregulated by pEGFP/N1-PCBP1, as shown in (Figures 6(D)-(F)).

We further identified that the indirect action of PCBP1 has a biological effect on the proteins of NF- $\kappa \mathrm{B}, \mathrm{HSP} 70, \mathrm{BDNF}$, and GDNF. Western blots and CLSM (green color due to Alexa Fluor 488 fluorophore) demonstrated that the supernatant from SH-SY5Y cell culture overexpressing PCBP1 significantly increased the expression level of HSP70 and repressed the expression level of NF- $\kappa \mathrm{B}$, while there was no statistically significant change in GDNF in BV-2 and HA cells (Figure 7). The supernatants from BV-2 and HA cell cultures overexpressing PCBP1 also significantly increased the expression level of HSP70 and repressed the expression level of NF- $\kappa \mathrm{B}$, whereas there was no statistically significant effect on the expression of BDNF in the SH-SY5Y cells (Figure 7). Therefore, PCBP1 upregulates the expression level of HSP70 and inhibits the level of NF- $\kappa \mathrm{B}$ in direct and indirect action models, whereas PCBP1 only upregulates the expression of GDNF and BDNF in direct action rather than in the indirect action model.

\section{Discussion}

PCBP1 has recently been shown to play a crucial role in the regulation of the mRNA of cancer metastasis, iron storage and virus replication [25] [26] [27] [28]. Due to the strong potential of PCBP1 to regulate many mRNAs, it is not surprising that PCBP1 also alters transcripts and translatants important for well-known neuropathy genes and neuronal processes. Increasing numbers of studies have revealed some neurous functions of PCBP1, including neuronal myelin formation and neuron cell apoptosis [29] [30]. These findings highlight the clinical significance of PCBP1 in neurous disease. Thus, investigating alternative PCBP1 function in neurons and glial cells would be helpful for neurous disease gene therapy. The direct and indirect regulation models of PCBP1 provide a rationale in assessing whether PCBP1 or productions of PCBP1 overexpression can display a neuroprotective effect.

Here, we capture PCBP1 overexpression by lipofectin transfection. Our results indicate that PCBP1 was overexpressed in SH-SY5Y, HA and BV-2 cells by western blot and real-time qPCR. In the present study, we demonstrate that PCBP1 overexpression stimulates the growth of SH-SY5Y, HA and BV-2 cells through direct actions, whereas the production of PCBP1 has no vital effect on the proliferation of SH-SY5Y, HA and BV-2 cells. Because we observe proliferation during the direct action with the neuronal cells, we conclude that PCBP1 needs to be expressed in the cells to have a positive effect. Apoptosis is a biological activity related to cell growth. However, by conducting an apoptosis assay, we also revealed that PCBP1 inhibits the early apoptosis of SH-SY5Y, HA and BV-2 cells in the direct action model, contrastingly having less effect on apoptosis in the indirect model. The influence of PCBP1 on apoptosis remains a contentious and controversial issue. For example, the PCBP1 KH1 domain binds to 


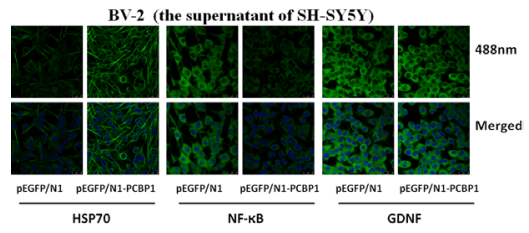

(A)

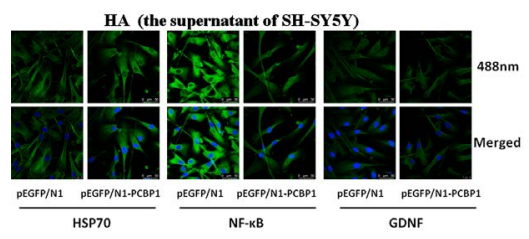

(B)

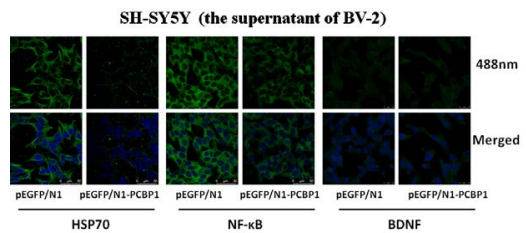

(C)

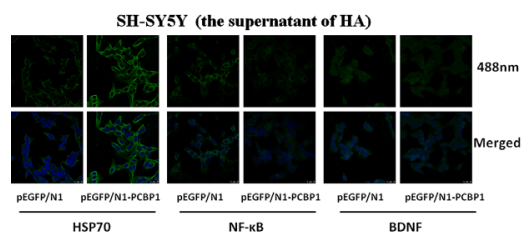

(D)

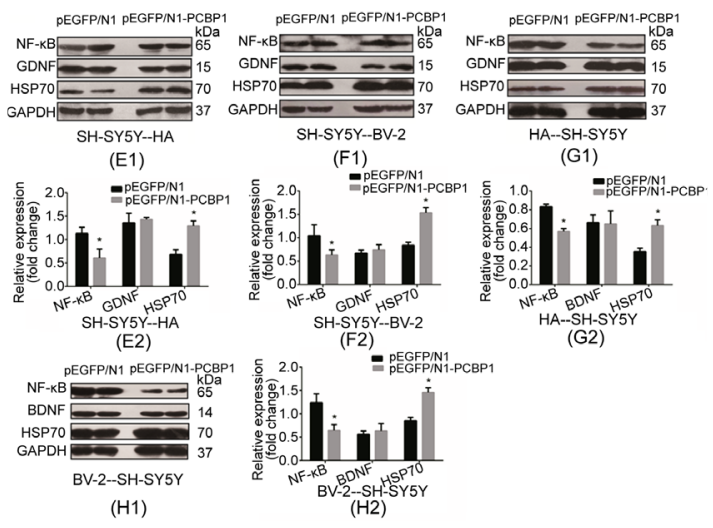

Figure 7. PCBP1 enhances the expression of HSP70, BDNF and GDNF and inhibits $\mathrm{NF}-\kappa \mathrm{B}$ signaling pathway activation by indirect effects. In the confocal images, the top row shows the fluorescence of HSP70, BDNF, GDNF and NF- $\kappa$ B, stained green, observed at $488 \mathrm{~nm}$. In the bottom row, the images of the top panel and nuclei that were stained blue (Hoechst 33,258) were merged to obtain a composite. ((A)-(D)) Confocal images show the increased fluorescence of HSP70 (green) and the decreased fluorescence of $\mathrm{NF}-\kappa \mathrm{B}$ (green), whereas there is no statistically significant change of GDNF and BDNF (green) in the indirect action model. (E-H) Western blot showed increased levels of HSP70 and a decline of NF- $\kappa$ B in SH-SY5Y--HA ((E1) and (E2)), SH-SY5Y--BV-2 ((F1) and (F2)), HA--SH-SY5Y ((G1) and (G2)), and BV-2--SH-SY5Y ((H1) and (H2)) cells with indirect action. ${ }^{*}$ Statistically significant differences $(\mathrm{p}<0.05)$ between $\mathrm{pEGFP} / \mathrm{N} 1$ and pEGFP/N1-PCBP1. GAPDH was probed as a protein loading control for all experiments. The bars express as mean \pm S.D., $\mathrm{n}=3$. 
P27 mRNA, leading to upregulated P27 levels and blockage of the S cell cycle, which promotes apoptosis [31]. In contrast, Waggoner et al. found that PCBP1 is significant in cell cycle progression: if PCBP1 genes are knocked out from K562 cells, cell proliferation is limited, and the cell cycle is blocked in the G1 cell cycle [8]. In addition, the mRNA levels of DFFB and MCL1, which are related to apoptosis, as well as BRBL1 and ABL1, which are related to the cell cycle, are increased by the overexpression of PCBP1 [32]. This increase indicates that PCBP1 has two-sided effects on apoptosis.

Aside from proliferation and anti-apoptosis, PCBP1 also contains other functions, such as trophic factor secretion and anti-inflammation, which could contribute to the observed protection in neuronal cells. Glial cell line-derived neurotrophic factor (GDNF) and brain-derived neurotrophic factor (BDNF) are neurotrophic factors that are involved in regulating neurite outgrowth, neuronal survival and synaptic activity [33] [34]. Kang et al. illuminated that NF- $\kappa$ B plays an important role in the $\mathrm{n}$ lipopolysaccharide (LPS)-induced activation of cyclooxygenase-2 (COX-2) in BV-2 cells [35]. Considering that the direct regulation of PCBP1 overexpression resulted in increased levels of mRNA expression of GDNF, BDNF and HSP70, as well as downregulated expression of NF- $\kappa \mathrm{B}$, we indicated how significant the expression of PCBP1 is for a healthy central nervous system. Our indirect action model indicated an increase in the expression of HSP70 and a decrease in the expression of NF- $\kappa \mathrm{B}$, while there was no obvious effect on the expression of GDNF and BDNF. It is further demonstrated that PCBP1 products may be the key to protecting the nervous system microenvironment. In line with this possibility, our previous study showed that the protein levels of HSPA6 and HSPA1A, which are members of the heat shock protein 70 (HSP70) family, were upregulated in PCBP1-overexpressing SH-SY5Y cells [32]. The synaptic deficits of the hippocampus in the HD mouse model were rescued by enhanced BDNF levels [36] [37]. GDNF exhibited significant antioxidant properties, which reduced cell death [38].

IL-10 is an immunoregulatory cytokine with various effects on the downregulation of chemokines, pro-inflammatory cytokines and costimulatory molecules [39] [40]. Our results unveiled that PCBP1 overexpression stimulated an increase in IL-10 expression and downregulation of IL- 6 by direct and indirect actions in BV-2 cells compared to the control group. However, we demonstrated that the mRNA level of IL-6 was overexpressed, whereas IL-10 expression was not statistically significant through the direct and indirect regulation of PCBP1 in SH-SY5Y and HA cells. In accordance with our observation, PCBP1 binding with STAT3C was involved in STAT3-IL-10-mediated suppression of IL-6 production and NF- $\kappa$ B activation [21]. Meanwhile, the upregulation of the dominant-negative form of STAT3 enhanced the expression of IL-10 to inhibit LPS-induced IL-6 and TNF- $\alpha$ production [41] [42]. Yi-Chin Lin et al. reported that IL-10 acted as a neuroprotective effect on inhibiting LPS-induced microglial activation [43]. Therefore, PCBP1 suppresses the production of proinflammatory IL- 6 by stimulating anti-inflammatory IL-10, which may be a partial signaling 
pathway for the observed neuroprotective effect of PCBP1. In summary, significant evidence from this manuscript reveals that PCBP1 is involved in the neuroprotective mechanism from direct and indirect regulation.

\section{Conclusion}

In conclusion, we report that the overexpression of PCBP1 and the substances secreted of PCBP1 overexpression in the cell regulate neuroblastoma cells and glial cells. In the study, empolying the direct and indirect model, it has been demonstrated that PCBP1 is involved in the neuroprotective mechanism, including proliferation, anti-apoptosis, anti-inflammation and the expression of functional proteins. Intriguingly, from a gene therapeutic point, Neurons and glial cells expressing PCBP1 might be more effective for nervous system damage, providing a novel and valid therapeutic opportunities for the study of neurological diseases through the overexpression of PCBP1.

\section{Acknowledgements}

The human neurblastoma cell line (SH-SY5Y) was generous gifts from Institute of Pediatrics, Beijing Children's Hospital, Capital Medical University. The human astrocyte cell line (HA) was generous gifts from Institute of Neurosurgery, Beijing Tiantan Hospital, Capital Medical University. The microglial cell line (BV-2) was generous gifts from Neurobiology Laboratory, Capital Medical University. This work was supported by National Natural Science Foundation of China (youth fund) (31200811), "Discipline backbone" project of high-level health technical personnel training program of Beijing health system (2013-3-096) and Xicheng District excellent talents top team project of Beijing (201915)

\section{Author Contributions}

LH designed the study, analysis of the data; BJ, acquisition and analysis of the data, drafted the manuscript; MY and CL revised the manuscript.

\section{Conflicts of Interest}

The authors declare that they have no conflicts of interest.

\section{References}

[1] Makeyev, A.V. and Liebhaber, S.A. (2002) The Poly (C)-Binding Proteins: A Multiplicity of Functions and a Search for Mechanisms. $R N A, \mathbf{8}, 265-278$. https://doi.org/10.1017/S1355838202024627

[2] Ren, C., Cho, S.J., Jung, Y.S. and Chen, X. (2014) DNA Polymerase $\eta$ Is Regulated by Poly (rC)-Binding Protein 1 via mRNA Stability. Biochemical Journal, 464, 377-386. https://doi.org/10.1042/BJ20141164

[3] Makeyev, A.V. and Liebhaber, S.A. (2000) Identification of Two Novel Mammalian Genes Establishes a Subfamily of KH-Domain RNA-Binding Proteins. Genomics, 67, 301-316. https://doi.org/10.1006/geno.2000.6244 
[4] Choi, H.S., Hwang, C.K., Song, K.Y., Law, P.-Y., Wei, L.-N. and Loh, H.H. (2009) Poly (C)-Binding Proteins as Transcriptional Regulators of Gene Expression. Biochemical and Biophysical Research Communications, 380, 431-436. https://doi.org/10.1016/j.bbrc.2009.01.136

[5] Ji, X., Wan, J., Vishnu, M., Xing, Y. and Liebhaber, S.A. (2013) aCP Poly (C) Binding Proteins Act as Global Regulators of Alternative Polyadenylation. Molecular and Cellular Biology, 33, 2560-2573. https://doi.org/10.1128/MCB.01380-12

[6] Chaudhury, A., Chander, P. and Howe, P.H. (2010) Heterogeneous Nuclear Ribonucleoproteins (hnRNPs) in Cellular Processes: Focus on hnRNP E1's Multifunctional Regulatory Roles. $R N A, 16,1449-1462$.

https://doi.org/10.1261/rna.2254110

[7] Ostareck-Lederer, A., Ostareck, D.H. and Hentze, M.W. (1998) Cytoplasmic Regulatory Functions of the KH-Domain Proteins hnRNPs K and E1/E2. Trends in Biochemical, 23, 409-411. https://doi.org/10.1016/S0968-0004(98)01301-2

[8] Waggoner, S.A., Johannes, G.J. and Liebhaber, S.A. (2009) Depletion of the Poly (C)-Binding Proteins alphaCP1 and alphaCP2 from K562 Cells Leads to p53-Independent Induction of Cyclin-Dependent Kinase Inhibitor (CDKN1A) and G1 Arrest. Journal of Biological Chemistry, 284, 9039-9049.

https://doi.org/10.1074/jbc.M806986200

[9] Ostareck-Lederer, A. and Ostareck, D.H. (2004) Control of mRNA Translation and Stability in Haematopoietic Cells: The Function of hnRNPs K and E1/E2. Biology of the Cell, 96, 407-411. https://doi.org/10.1016/j.biolcel.2004.03.010

[10] Zhang, T., Huang, X.H., Dong, L., Hu, D.Q., Ge, C.H., Zhan, Y.-Q., et al. (2010) PCBP-1 Regulates Alternative Splicing of the CD44 Gene and Inhibits Invasion in Human Hepatoma Cell Line HepG2 Cells. Molecular Cancer, 9, Article No. 72. https://doi.org/10.1186/1476-4598-9-72

[11] Liu, Y., Gai, L., Liu, J., Cui, Y., Cui, Y., Zhang, Y. and Feng, J. (2015) Expression of Poly (C)-Binding Protein 1 (PCBP1) in NSCLC as a Negative Regulator of EMT and Its Clinical Value. International Journal of Clinical and Experimental Pathology, 8, 7165-7172.

[12] Zhang, M.P., Zhang, W.S., Tan, J., Zhao, M.-H., Lian, L.-J. and Cai, J. (2017) Poly r (C) Binding Protein (PCBP $)_{1}$ Expression Is Regulated by the $\mathrm{E}_{3}$ Ligase UBE4A in Thyroid Carcinoma. Bioscience Reports, 37, 708-725. https://doi.org/10.1042/BSR20170114

[13] Chen, Q., Cai, Z.K., Chen, Y.B., Zheng, D., Zhou, J. and Wang, Z. (2015) Poly r (C) Binding Protein-1 Is Central to Maintenance of Cancer Stem Cells in Prostate Cancer Cells. Cellular Physiology and Biochemistry, 35, 1052-1061. https://doi.org/10.1159/000373931

[14] Shi, H., Bencze, K.Z., Stemmler, T.L. and Philpott, C.C. (2008) A Cytosolic Iron Chaperone That Delivers Iron to Ferritin. Science, 320, 1207-1210. https://doi.org/10.1126/science.1157643

[15] Nandal, A., Ruiz, J.C., Subramanian, P., Sinnamon, R.A., Stemmler, T.L., Bruick, R.K., et al. (2011) Activation of the HIF Prolyl Hydroxylase by the Iron Chaperones PCBP1 and PCBP2. Cell Metabolism, 14, 647-657. https://doi.org/10.1016/j.cmet.2011.08.015

[16] Frey, A.G., Nandal, A., Park, J.H., Smith, P.M., Yabe, T., Ryu, M.-S., et al. (2014) Iron Chaperones PCBP1 and PCBP2 Mediate the Metallation of the Dinuclear Iron Enzyme Deoxyhypusine Hydroxylase. Proceedings of the National Academy of Sciences of the United States of America, 111, 8031-8036. 
https://doi.org/10.1073/pnas.1402732111

[17] Dinh, P.X., Beura, L.K., Panda, D., Das, A. and Pattnaik, A.K. (2011) Antagonistic Effects of Cellular Poly (C) Binding Proteins on Vesicular Stomatitis Virus Gene Expression. Journal of Virology, 85, 9459-9471. https://doi.org/10.1128/JVI.05179-11

[18] Makarov, S.S. (2000) NF-kappaB as a Therapeutic Target in Chronic Inflammation: Recent Advances. Trend in Molecular Medicine, 6, 441-448. https://doi.org/10.1016/S1357-4310(00)01814-1

[19] Mattson, M.P. and Camandola, S. (2001) NF-kappaB in Neuronal Plasticity and Neurodegenerative Disorders. The Journal of Clinical Investigation, 107, 247-254. https://doi.org/10.1172/JCI11916

[20] Hunot, S., Brugg, B., Ricard, D., Michel, P.P., Muriel, M.-P., Ruberg, M., et al. (1997) Nuclear Translocation of NF-kappaB Is Increased in Dopaminergic Neurons of Patients with Parkinson Disease. Proceedings of the National Academy of Sciences of the United States of America, 94, 7531-7536.

https://doi.org/10.1073/pnas.94.14.7531

[21] Nishinakamura, H., Minoda, Y., Saeki, K., Koga, K., Takaesu, G., Onodera, M., et al. (2007) An RNA-Binding Protein alphaCP-1 Is Involved in the STAT3-Mediated Suppression of NF-kappaB Transcriptional Activity. International Immunology, 19, 609-619. https://doi.org/10.1093/intimm/dxm026

[22] Zhong, N., Radu, G., Ju, W. and Ted Brown, W. (2005) Novel Progerin-Interactive Partner Proteins hnRNP E1, EGF, Mel 18, and UBC9 Interact with Lamin A/C. Biochemical and Biophysical Research Communications, 338, 855-861. https://doi.org/10.1016/j.bbrc.2005.10.020

[23] Geuens, T., De Winter, V., Rajan, N., Achsel, T., Mateiu, L., Almeida-Souza, L., et al. (2017) Mutant HSPB1 Causes Loss of Translational Repression by Binding to PCBP1, an RNA Binding Protein with a Possible Role in Neurodegenerative Disease. Acta Neuropathologica Communications, 5, Article No. 5. https://doi.org/10.1186/s40478-016-0407-3

[24] Huo, L.R., Ju, W., Yan, M., Zou, J.-H., Yan, W., He, B., et al. (2010) Identification of Differentially Expressed Transcripts and Translatants Targeted by Knock-Down of Endogenous PCBP1. Biochimica et Biophysica Acta (BBA)-Proteins and Proteomics, 1804, 1954-1964. https://doi.org/10.1016/j.bbapap.2010.07.002

[25] Giles, K.M., Daly, J.M., Beveridge, D.J., Thomson, A.M., Voon, D.C., Furneaux, H.M., et al. (2003) The 3'-Untranslated Region of p21WAF1 mRNA Is a Composite Cis-Acting Sequence Bound by RNA-Binding Proteins from Breast Cancer Cells, Including HuR and Poly (C)-Binding Protein. Journal of Biological Chemistry, 278, 2937-2946. https://doi.org/10.1074/jbc.M208439200

[26] Wang, H., Vardy, L.A., Tan, C.P., Loo, J.M., Guo, K., Li, J., et al. (2010) PCBP1 Suppresses the Translation of Metastasis-Associated PRL-3 Phosphatase. Cancer Cell, 18, 52-62. https://doi.org/10.1016/j.ccr.2010.04.028

[27] Leidgens, S., Bullough, K.Z., Shi, H., Li, F.M., Shakoury-Elizeh, M., Yabe, T., et al. (2013) Each Member of the Poly-r (C)-Binding Protein 1 (PCBP) Family Exhibits Iron Chaperone Activity toward Ferritin. Journal of Biological Chemistry, 288, 17791-17802. https://doi.org/10.1074/jbc.M113.460253

[28] Zhou, X., You, F., Chen, H. and Jiang, Z. (2012) Poly (C)-Binding Protein 1 (PCBP1) Mediates Housekeeping Degradation of Mitochondrial Antiviral Signaling (MAVS). Cell Research, 22, 717-727. https://doi.org/10.1038/cr.2011.184

[29] Thyagarajan, A. and Szaro, B.G. (2004) Phylogenetically Conserved Binding of Spe- 
cific K Homology Domain Proteins to the 3'-Untranslated Region of the Vertebrate Middle Neurofilament mRNA. Journal of Biological Chemistry, 279, 49680-49688. https://doi.org/10.1074/jbc.M408915200

[30] Thyagarajan, A. and Szaro, B.G. (2008) Dynamic Endogenous Association of Neurofilament mRNAs with K-Homology Domain Ribonucleoproteins in Developing Cerebral Cortex. Brain Research, 1189, 33-42. https://doi.org/10.1016/j.brainres.2007.11.012

[31] Shi, H., Li, H., Yuan, R., Guan, W., Zhang, X.M., Zhang, S.Y., et al. (2018) PCBP1 Depletion Promotes Tumorigenesis through Attenuation of p27 ${ }^{\text {Kip } 1}$ mRNA Stability and Translation. Journal of Experimental \& Clinical Cancer Research, 37, Article No. 187. https://doi.org/10.1186/s13046-018-0840-1

[32] Huo, L.R. and Zhong, N. (2008) Identification of Transcripts and Translatants Targeted by Overexpressed PCBP1. Biochimica et Biophysica Acta (BBA)-Proteins and Proteomics, 1784, 1524-1533. https://doi.org/10.1016/j.bbapap.2008.06.017

[33] Lin, L.F., Doherty, D.H., Lile, J.D., Bektesh, S. and Collins, F. (1993) GDNF: A Glial Cell Line-Derived Neurotrophic Factor for Midbrain Dopaminergic Neurons. Science, 260, 1130-1132. https://doi.org/10.1126/science.8493557

[34] Park, H. and Poo, M.M. (2013) Neurotrophin Regulation of Neural Circuit Development and Function. Nature Reviews Neuroscience, 14, 7-23. https://doi.org/10.1038/nrn3379

[35] Kang, S.M., More, S.V., Park, J.Y., Kim, B.-W., Park, J.I. and Yoon, S.-H. (2014) A Novel Synthetic HTB Derivative, BECT Inhibits Lipopolysaccharide-Mediated Inflammatory Response by Suppressing the p38 MAPK/JNK and NF- $\kappa$ B Activation Pathways. Pharmacological Reports, 66, 471-479.

https://doi.org/10.1016/j.pharep.2013.08.015

[36] Lynch, G., Kramar, E.A., Rex, C.S., Jia, Y.S., Chappas, D., Gall, C.M., et al. (2007) Brain-Derived Neurotrophic Factor Restores Synaptic Plasticity in a Knock-in Mouse Model of Huntington's Disease. Journal of Neuroscience, 27, 4424-4434. https://doi.org/10.1523/JNEUROSCI.5113-06.2007

[37] Brito, V., Giralt, A., Enriquez-Barreto, L., Puigdellívol, M., Puigdellívol, M., Suelves, N., Zamora-Moratalla, A., et al. (2014) Neurotrophin Receptor p75 (NTR) Mediates Huntington's Disease-Associated Synaptic and Memory Dysfunction. Journal of Clinical Investigation, 124, 4411-4428. https://doi.org/10.1172/JCI74809

[38] Mishchenko, T.A., Mitroshina, E.V., Shishkina, T.V. and Vedunova, M.B. (2018) Antioxidant Properties of Glial Cell-Derived Neurotrophic Factor (GDNF). Bulletin of Experimental Biology and Medicine, 166, 293-296. https://doi.org/10.1007/s10517-018-4335-Z

[39] Saraiva, M. and O'Garra, A. (2010) The Regulation of IL-10 Production by Immune Cells. Nature Reviews Immunology, 10, 170-181. https://doi.org/10.1038/nri2711

[40] de Oca, M.M., Kumar, R., de Labastida Rivera, F., et al. (2016) Blimp-1-Dependent IL-10 Production by Tr1 Cells Regulates TNF-Mediated Tissue Pathology. PLoS Pathog, 12, e1005398. https://doi.org/10.1371/journal.ppat.1005460

[41] Rajasingh, J., Bord, E., Luedemann, C., Asai, J., Hamada, H., Thorne, T., et al. (2006) IL-10-Induced TNF-alpha mRNA Destabilization is Mediated via IL-10 Suppression of p38 MAP Kinase Activation and Inhibition of HuR Expression. The FASEB Journal, 20, 2112-2114. https://doi.org/10.1096/fj.06-6084fje

[42] Williams, L., Bradley, L., Smith, A. and Foxwell, B. (2004) Signal Transducer and Activator of Transcription 3 Is the Dominant Mediator of the Anti-Inflammatory Effects of IL-10 in Human Macrophages. The Journal of Immunology, 172, 567-576. 
https://doi.org/10.4049/jimmunol.172.1.567

[43] Lin, Y.C., Uang, H.W., Lin, R.J., Chen, I.-J. and Lo, Y.-C. (2007) Neuroprotective Effects of Glyceryl Nonivamide against Microglia-Like Cells and 6-Hydroxydopamine-Induced Neurotoxicity in SH-SY5Y Human Dopaminergic Neuroblastoma Cells. Journal of Pharmacology and Experimental Therapeutics, 323, 877-887. https://doi.org/10.1124/jpet.107.125955 